\title{
Comparison of Outcomes of Community and Hospital-Ac- Quired Methicillin- Susceptible and Methicillin-Resistant Staphylococcus Aureus Infections
}

\author{
Research Article
}

Volume 2 Issue 1- 2021

\author{
Author Details \\ Zied Gaifer ${ }^{1 *}$, Talha Youssouf ${ }^{1}$, Basmah H Aljehani², Abdulkader Ismail ${ }^{3}$, Ahmed Abdelaziz ${ }^{3}$ \\ ${ }^{1}$ Department of Medicine, National Guard Hospital, Saudi Arabia \\ ${ }^{2}$ Department of Infection Prevention and Control, National Guard Hospital, Saudi Arabia \\ ${ }^{3}$ Department of Laboratory Medicine, National Guard Hospital, Saudi Arabia \\ *Corresponding author
}

Zied Gaifer Ali, Prince Mohmed Bin Abdulaziz hospital, National Guard Health affair, Department of Medicine, Medina 41511, Saudi Arabia

Article History

Received: December 12, 2020 Accepted: April 20, 2021 Published: April 22, 2021

\begin{abstract}
Introduction: Staphylococcus Aureus (S. aureus) causes community (CA) and hospital-acquired (HA) infections that kills millions of patients every year. It is unclear if Methicillin-resistant Staphylococcus Aureus (MRSA) have higher mortality and complication than Methicillin -susceptible Staphylococcus Aureus (MSSA) infections in CA and HA infections.

Methods: This retrospective study included confirmed of cases S. aureus infections, which classified as MSSA or MRSA and CA or HA infections We assessed 30-day mortality, rate of Septic Shock and Acute Renal Injury (AKI) as outcomes measures.

Results: Of the total 183 cases included, we found no differences between MRSA and MSSA cases in mortality $(\mathrm{P}=0.734)$ and other outcomes. Likewise, CA and HA cases Similar mortality. However, HA cases developed a higher rate of AKI compared to CA cases. We observed this increased rate of AKI only in HA MSSA cases.

Conclusion: This study offers a new perspective on HA MSSA as a serious pathogen as MRSA, that requires intervention to prevent its spread. Future research needed to identify new measures for MSSA prevention and investigate whether current MRSA prevention strategies are effective in MSSA.
\end{abstract}

Keywords: Methicillin-resistant; MRSA; MSSA; Saudi Arabia; Staphylococcus aureus

\section{Introduction}

Staphylococcus Aureus (S. aureus) is the commonest bacteria that causes infection inside and outside the hospitals. It is accountable for millions of deaths every year worldwide [1]. Treatment of $S$. aureus became more difficult and expensive due to the increasing rate of methicillin-resistant strains, which are now responsible for many hospital and community-acquired infections $[2,3]$.

Studies have suggested that methicillin resistance and the location of acquiring $S$. aureus infection are the main determinants of its outcome. Still, it is controversial whether Methicillin-resistant Staphylococcus Aureus (MRSA) causes a worse outcome compared to Methicillin-sus- ceptible Staphylococcus Aureus (MSSA) infections [4-7]. Another area of debate, whether hospital-acquired S. aureus (HA-S. aureus) has a worse outcome than community-acquired S. aureus (CA- S. aureus) infections [8-10]. Taken together, it remains an open question whether the setting of acquiring $S$. aureus infection and methicillin susceptibility are the main determinants of its outcomes.

In this study, we explored the outcomes of $S$. aureus infection and we adjusted for patients' comorbidities. The research question of the present article is whether hospital-acquired MSSA infection causes more morbidity and mortality than community-acquired MSSA infections. We also compared the mortality of HA-MRSA to CA-MRSA infections and MRSA to MSSA infections. 


\section{Methods and Materials Subjects}

We conducted this retrospective observational study between January 2017 and February 2018, in National Guard Hospital in Al-Madinah in Saudi Arabia. We included 15 years and older patients, who had a documented diagnosis of $S$. aureus infection in the electronic medical records during the study period. The diagnosis of $S$. aureus infection was verified by the existence of a positive culture of $S$. aureus from the infected site and documented signs and symptoms of infection. Institutional review board approved the study.

\section{Microbiologic methods}

S. aureus cultures were processed by BAC T/ALERT, version eight (BioMerieux). Antimicrobial susceptibility testing was performed by Broth micro-dilution method using VITEK 2, version eight (BioMerieux). S. aureus susceptibility breakpoints defined according to the Clinical and Laboratory Standards Institute (CLSI) breakpoints [11].

\section{Definitions and criteria}

We classified infections as community-acquired S. aureus infections if the infection acquired in the community and within the last 3 months there were no history of hospitalization, surgery, residence in a longterm care facility, dialysis, presence of invasive medical devices or previous isolation of $S$. aureus [12]. Otherwise, the infections were classified as hospital-acquired. Outcome measures assessed were thirty-day all-cause mortality, duration of Intensive Care Unit (ICU) stay, rate of septic shock and rate of Acute Renal Injury (AKI).

The severity of $S$. aureus infection measured using the Sequential Organ Failure Assessment (SOFA) score and Acute Physiologic
Assessment and Chronic Health Evaluation (APACHE II score). We used Charlson's comorbidity index to measure concurrent comorbidities [13].

\section{Data analysis}

Mean, the median, and the Standard Deviation (SD) calculated for continuous variables. We calculated proportions for categorical variables. Chi square, Fisher exact test and Logistic regression were used to compare categorical variables. Student t-test was used to compare continues normally distributed data and Wilcoxon RankSum used to compare non-parametric variables. Multivariable logistic regression analysis was used to estimate the effect of risk factors on primary outcomes, to control for confounding and to assess for interaction. All P-values were two-tailed, and values of $\leq 0.05$ were considered statistically significant. Analysis was performed using STATA version 13 (STATA cooperation, Texas, USA).

\section{Results}

\section{MRSA compared to MSSA infections}

This study reviewed 183 patients with invasive S. aureus infections from January 2017 to February 2018. First, we compared the outcome of MRSA with MSSA infections using Chi square and Fisher exact test (Table 1). The median age was 45 years, and the cases were predominately males (58\%). Skin and soft tissue were the main infection sites (71.58\%). Of all cases, 18 (9.84\%) developed septic shock and $9(4.92 \%)$ died. Of all S. aureus isolates, MRSA was recovered in $84(45.9 \%)$ cases. There were no significant differences between MRSA and MSSA infections regarding bacteremia $(\mathrm{P}=0.686)$, days of ICU stay $(\mathrm{P}=0.422)$, septic shock $(\mathrm{P}=0.999)$ and 30 -day mortality $(\mathrm{P}=0.734)$.

Table 1: Baseline characteristics of patients with invasive Staphylococcus Aureus infections (January 2017- February 2018).

\begin{tabular}{|c|c|c|c|c|}
\hline \multirow{3}{*}{ Characteristics } & Total & MRSA & MSSA & \multirow{3}{*}{ P-value } \\
\hline & n/(N \%) & n/(N \%) & n/(N \%) & \\
\hline & $183(100 \%)$ & $84(45.90)$ & $99(54.10)$ & \\
\hline \multicolumn{5}{|c|}{ Age (Year) } \\
\hline Mean (SD) & $43(28)$ & $44(28)$ & $42(29)$ & 0.448 \\
\hline Median (IQR) & $45(17-68)$ & $43(25-68)$ & $47(9-66)$ & \\
\hline Male & 107(58.47) & $48(57.14)$ & $59(59.60)$ & 0.765 \\
\hline Bacteremia & $29(15.85)$ & $12(14.29)$ & $17(17.17)$ & 0.686 \\
\hline Thirty-day mortality & $9(4.92)$ & $5(5.95)$ & $4(4.04)$ & 0.734 \\
\hline \multicolumn{5}{|c|}{ Site of the Infection } \\
\hline Pneumonia & $19(10.38)$ & $10(11.90)$ & $9(9.09)$ & 0.629 \\
\hline Soft tissue infection & $131(71.58)$ & $57(67.86)$ & $74(56.49)$ & 0.327 \\
\hline Bone and joint infections & $14(7.65)$ & $8(9.52)$ & $6(6.06)$ & 0.415 \\
\hline Endocarditis & $2(1.09)$ & $2(2.38)$ & $0(0.00)$ & 0.209 \\
\hline Community- acquired & $127(69.40)$ & $55(65.48)$ & $72(72.73)$ & 0.335 \\
\hline Days of ICU stay (mean) & $4.94(37)$ & $7(53)$ & $3(13)$ & 0.422 \\
\hline \multicolumn{5}{|c|}{ Severity of Sepsis } \\
\hline Sepsis & $75(40.98)$ & $35(41.67)$ & $40(40.40)$ & 0.881 \\
\hline Severe sepsis & $15(8.20)$ & $5(5.95)$ & $10(10.10)$ & 0.42 \\
\hline Septic shock & $18(9.84)$ & $8(9.52)$ & $10(10.10)$ & 1 \\
\hline Sofa score (mean) & $3.85(1.64)$ & $3.66(1.6)$ & $4(1.66)$ & 0.174 \\
\hline Sofa score (median) & $3(3-4)$ & $3(3-4)$ & $3(3-4)$ & - \\
\hline Acute renal failure & $25(13.66)$ & $9(10.71)$ & $16(16.16)$ & 0.388 \\
\hline
\end{tabular}




\begin{tabular}{|c|c|c|c|c|}
\hline \multicolumn{7}{|c|}{ Apache II Score } \\
\hline Mean & $8.2(7.7)$ & $7.0(6.8)$ & $9.3(8.3)$ & 0.051 \\
\hline Median & $6(3-12)$ & $5(2-11)$ & $7(4-14)$ & - \\
\hline \multicolumn{7}{|c|}{ Charlson Comorbidity Index } & $1.86(2.8)$ & 0.437 \\
\hline Mean & $1.71(2.7)$ & $1.5(2.7)$ & $0(0-3)$ & - \\
\hline
\end{tabular}

MSSA: Methicillin-Susceptible Staphylococcus Aureus; MRSA: Methicillin-Resistant Staphylococcus Aureus; SOFA: Sequential (sepsis-related) Organ Failure Assessment; APACHE II score: Acute Physiologic Assessment and Chronic Health Evaluation; ICU: Intensive Care Unit; P value significant if <0.05

\section{Hospital-acquired compared community S. aureus infections}

Second, we compared the outcome of $S$. aureus hospital-acquired infection (HA S. aureus) with $S$. aureus community (CA S. aureus) infections. Of the total cases $56(30 \%)$ were classified as HA $S$. aureus (Table 2). Of the those 29 (51.79\%) cases were HA-MRSA. of the total CA S. aureus 55(43.31) were MRSA. In crude analysis, HA S. aureus infections had a higher rate of septic shock [odd ratio (OR) 4.19, 95\% confidence interval (CI) ([1.529-11.48]; $\mathrm{p}<0.005)]$,AKI (OR $5.24,95 \%$ CI [2.15-12.79]; $\mathrm{p}<0.001$ ) and higher 30-day mortality (OR 4.96 ,CI 95\% [1.19-20.61]; $\mathrm{p}<0.028$ ) compared to CA S. aureus cases .In multivariable logistic regression analysis, we included the above significant variables and adjusted for chronic comorbidities and severity of illness. The only variable remained significant was AKI (adjusted OR 5.47 CI 95\% [1.51-19.90]; $\mathrm{p}<0.010$ ). This model also fitted with and without controlling for DM, and the above result remains unchanged.
In subgroup analysis we compared HA-MSSA and CA-MSSA to investigate further the above finding of a higher rate of acute renal failure in HA S. aureus compared to CA S. aureus infections. Of the total MSSA infections, 27 (27.27\%) cases were hospital acquired (Table 3 ). In the unadjusted analysis, factors that significantly associated with HA-MSSA compared to CA-MSSA were the ICU days (OR 1.21,95\% CI [1.02-1.43]; $\mathrm{P}<0.033$ ) septic shock (OR 8.05 ,95\%CI [1.91-34.012]; $\mathrm{P}<0.005)$ AKI (OR 9.21,95\%CI [2.80-30.27]; $\mathrm{P}<0.001)$. In adjusted logistic regression we included the above significant variables, and we adjusted for chronic comorbidities and severity of illness using Charlson comorbidity and APACHE II scores. HA-MSSA infections had higher rates of AKI (adjusted OR 13.80 [2.81-67.83]; $\mathrm{p}<0.001$ ) compared to CA-MSSA.

In subgroup analysis of MRSA infections (Table 4). We found no significant differences between HA-MRSA and CA-MRSA regarding rates of septic shock $(\mathrm{P}<0.438)$, mean days of ICU stay $(\mathrm{P}<0.327)$ and thirty-day mortality $(\mathrm{P}<0.999)$.

Table 2: Demographic Characteristics of Hospital-Acquired and Community-Acquired Staphylococcus Aureus infections cases.

\begin{tabular}{|c|c|c|c|c|}
\hline \multirow{2}{*}{ Characteristics } & \multirow{2}{*}{ All } & HA - S. aureus & CA - S. aureus & \multirow{2}{*}{ P-value } \\
\hline & & $56(30.6 \%)$ & $127(69.39 \%)$ & \\
\hline Age (mean) & $43.7(28)$ & $46(29)$ & $42(28)$ & 0.3216 \\
\hline Age (median) & $45.5(17-68)$ & $50(24-73)$ & $44.5(16-65)$ & - \\
\hline Male & 107(58.47) & $33(58.9)$ & $74(58.27)$ & 0.999 \\
\hline \multicolumn{5}{|c|}{ Site of the Infection } \\
\hline Bacteremia & $29(15.85)$ & $12(21.43)$ & $17(13.39)$ & 0.19 \\
\hline Pneumonia & $19(10.38)$ & $9(16.07)$ & $10(7.87)$ & 0.116 \\
\hline Soft tissue infection & $131(71.58)$ & $35(62.50)$ & $96(75.59)$ & 0.078 \\
\hline Bone and joint infections & $14(7.65)$ & $4(7.14)$ & $10(7.65)$ & 1 \\
\hline Endocarditis & $2(1.09)$ & $1(1.79)$ & $1(0.79)$ & 0.52 \\
\hline Thirty-day mortality & $9(4.92)$ & $6(10.71)$ & $3(2.36)$ & 0.028 \\
\hline Days of ICU (mean) & $4(37)$ & $6.46(19)$ & $4.2(43)$ & 0.721 \\
\hline Sepsis & $75(40.98)$ & $24(42.86)$ & $51(40.98)$ & 0.747 \\
\hline Severe sepsis & $15(8.20)$ & $4(7.14)$ & $11(8.66)$ & 1 \\
\hline Septic shock & $18(9.84)$ & $11(19.64)$ & $7(5.51)$ & 0.005 \\
\hline \multicolumn{5}{|c|}{ Sofa Score } \\
\hline Mean & $3.84(1.6)$ & $4.08(2.02)$ & $3.73(1.44)$ & 0.5053 \\
\hline Median & $3(3-4)$ & $3(3-5)$ & $3(3-4)$ & - \\
\hline Acute Renal failure & $25(13.66)$ & $16(28.57)$ & $9(7.09)$ & 0.001 \\
\hline MRSA Isolates & $84(45.90)$ & $29(51.79)$ & $55(43.31)$ & 0.335 \\
\hline MSSA Isolates & $99(54.10)$ & $27(48.21)$ & $72(56.69)$ & 0.335 \\
\hline \multicolumn{5}{|c|}{ APACHE II Score } \\
\hline Mean & $8.2(7.7)$ & $9.69(8.0)$ & $7.67(7.5)$ & 0.1616 \\
\hline Median & $6(3-12)$ & $9(2.5-16)$ & $6(3-11)$ & - \\
\hline
\end{tabular}




\begin{tabular}{|c|c|c|c|c|}
\hline \multicolumn{5}{|c|}{ Charlson Comorbidity } \\
\hline DM & & & & \\
\hline Mean & $1.17(2.79)$ & $2.17(2.66)$ & $1.50(2.83)$ & 0.139 \\
\hline Median & $0(0-3)$ & $1(0-4)$ & $0(0-2)$ & - \\
\hline
\end{tabular}

CA: Community-Acquired; HA: Hospital-Acquired; MSSA: Methicillin-Susceptible Staphylococcus Aureus; MRSA: Methicillin- Resistant Staphylococcus Aureus; SOFA: Sequential (sepsis-related) Organ Failure Assessment; APACHE II score: Acute Physiologic Assessment and Chronic Health Evaluation; ICU: Intensive Care Unit; P value significant if $<0.05$

Table 3: Demographic characteristics of hospital and community-acquired methicillin-sensitive Staphylococcus Aureus infections.

\begin{tabular}{|c|c|c|c|c|}
\hline \multirow{3}{*}{ Characteristics } & Total MSSA & HA- MSSA & CA-MSSA & \multirow{3}{*}{ P value } \\
\hline & n /N(\%) & n / N(\%) & n / N(\%) & \\
\hline & $99(100 \%)$ & $27(27.27)$ & $72(72.73)$ & \\
\hline \multicolumn{5}{|c|}{ Age (Years) } \\
\hline Mean & $42(29)$ & $43(28.66)$ & $42(38)$ & 0.814 \\
\hline Median & $47(9-66)$ & $52(15-66)$ & $46(7-68)$ & - \\
\hline Male & $59(59.60)$ & $15(55.56)$ & $44(61.11)$ & 0.651 \\
\hline \multicolumn{5}{|c|}{ Site of the Infection } \\
\hline Bacteremia & $17(17.17)$ & $8(29.63)$ & $9(12.50)$ & 0.07 \\
\hline Pneumonia & $9(9.09)$ & $4(14.81)$ & $5(6.94)$ & 0.251 \\
\hline Soft tissue infection & $74(74.75)$ & $17(62.96)$ & $57(79.17)$ & 0.121 \\
\hline Bone and joint infection & $6(6.06)$ & $3(11.11)$ & $3(4.17)$ & 0.341 \\
\hline Thirty- days mortality & $4(4.04)$ & $2(7.41)$ & $2(2.78)$ & 0.299 \\
\hline Length of ICU (mean) days & $3(13)$ & $10(25)$ & $0.347(1.48)$ & 0.033 \\
\hline \multicolumn{5}{|c|}{ Severity of Sepsis } \\
\hline Sepsis & $40(40.40)$ & 13(48.15) & $27(37.50)$ & 0.365 \\
\hline Severe sepsis & $10(10.10)$ & $1(3.70)$ & $9(12.50)$ & 0.278 \\
\hline Septic shock & $10(10.10)$ & $7(25.93)$ & $3(4.17)$ & 0.005 \\
\hline \multicolumn{5}{|c|}{ Charlson Comorbidity Index } \\
\hline Mean & $1.85(2.8)$ & $2.51(2.56)$ & $1.611(2.88)$ & 0.176 \\
\hline Median & $0(0-3)$ & $2(0-5)$ & $0(0-2.5)$ & - \\
\hline \multicolumn{5}{|l|}{ Sofa Score } \\
\hline Mean & $4(1.66)$ & $4.4(1.98)$ & $3.84(1.50)$ & 0.2913 \\
\hline Median & $3(3-4)$ & $4(3-6)$ & $3(3-4)$ & - \\
\hline Acute Renal Failure & $16(16.16)$ & $11(40.74)$ & $5(6.94)$ & 0.001 \\
\hline \multicolumn{5}{|c|}{ APACHE II Score } \\
\hline Mean & $9.34(8.32)$ & $11(8)$ & $8.7(8.39)$ & 0.233 \\
\hline Median & $7(4-14)$ & $11(3-16)$ & $6(5-12)$ & - \\
\hline
\end{tabular}

CA: Community-Acquired; HA: Hospital-Acquired; MSSA: Methicillin-Susceptible Staphylococcus Aureus; MRSA: Methicillin- Resistant Staphylococcus Aureus; SOFA Sequential (sepsis-related) Organ Failure Assessment

Table 4: Hospital and community acquired methicillin- resistant Staphylococcus Aureus infections (January 2017- February 2018).

\begin{tabular}{|c|c|c|c|c|}
\hline \multirow{2}{*}{ Characteristics } & All MRSA & HA-MRSA & CA- MRSA & \multirow{2}{*}{ P value } \\
\cline { 2 - 4 } & $\mathbf{n} /(\mathbf{N} \%)$ & $\mathbf{n} /(\mathbf{N} \%)$ & $\mathbf{n} /(\mathbf{N} \%)$ & \\
\cline { 2 - 4 } & $\mathbf{8 4}(\mathbf{1 0 0} \%)$ & $\mathbf{2 9 ( 3 4 . 5 2 )}$ & $\mathbf{5 5}(\mathbf{6 5 . 4 8})$ & \\
\hline \multicolumn{5}{|c|}{ Age (Years) } \\
\hline Mean & $44(28.12)$ & $49(29.5)$ & $42(27.29)$ & 0.2834 \\
\hline Median & $43(25.5-68.5)$ & $43(32-77)$ & $44(17-65)$ & - \\
\hline Male & $48(57.14)$ & $18(62.07)$ & $30(54.55)$ & 0.644 \\
\hline Bacteremia & $12(14.29)$ & $4(13.79)$ & $8(14.55)$ & 1 \\
\hline Thirty-days mortality & $5(5.95)$ & $4(13.79)$ & $1(1.82)$ & 0.999 \\
\hline
\end{tabular}




\begin{tabular}{|c|c|c|c|c|}
\hline \multicolumn{5}{|c|}{ Site of the Infection } \\
\hline Pneumonia & $10(11.90)$ & $5(17.24)$ & $5(9.09)$ & 0.303 \\
\hline Soft tissue infection & $57(67.86)$ & $18(62.07)$ & $39(70.91)$ & 0.466 \\
\hline Bone and joint infection & $8(9.52)$ & $1(3.45)$ & $7(3.45)$ & 0.253 \\
\hline Endocarditis & $2(2.38)$ & $1(3.45)$ & $1(1.82)$ & 1 \\
\hline Days ICU(mean) days & $7(53)$ & $3(10)$ & $9(65)$ & 0.327 \\
\hline \multicolumn{5}{|l|}{ Severity of Sepsis } \\
\hline Sepsis & $35(41.67)$ & $11(37.93)$ & $24(43.64)$ & 0.649 \\
\hline Severe sepsis & $5(5.95)$ & $3(10.34)$ & $2(3.64)$ & 0.335 \\
\hline Septic shock & $8(9.52)$ & $4(13.79)$ & $4(7.27)$ & 0.438 \\
\hline Acute renal failure & $9(10.71)$ & $5(17.24)$ & $4(7.27)$ & 0.264 \\
\hline \multicolumn{5}{|l|}{ APACHE II Score } \\
\hline Mean & $7.04(6.8)$ & $8.44(8)$ & $6.29(6)$ & 0.863 \\
\hline Median & $5(2-11)$ & $6(2-12)$ & $5(2-8)$ & - \\
\hline \multicolumn{5}{|l|}{ Sofa Score } \\
\hline Mean & $3.66(1.6)$ & $3.79(2.04)$ & $3.59(1.36)$ & 0.9255 \\
\hline Median & $3(3-4)$ & $3(3-5)$ & $3(3-4)$ & - \\
\hline \multicolumn{5}{|l|}{ Charlson Comorbidity } \\
\hline Mean & $1.53(2.78)$ & $1.86(2.76)$ & $1.36(2.80)$ & 0.157 \\
\hline Median & $0(0-2)$ & $0(0-3)$ & $0(0-2)$ & - \\
\hline
\end{tabular}

MRSA: Methicillin-resistant Staphylococcus Aureus; DM: Diabetes Mellitus; PVD: Peripheral Vascular Disease; MSSA: Methicillin-Sensitive Staphylococcus Aureus; CA: Community acquired; HA: Hospital acquired; $P$ value significant if $<0.05$

\section{Discussion}

This study investigated the outcomes of community (HA) and hospitalacquired (CA) S. aureus infections, and it explored the impact of methicillin susceptibility on these outcomes. We found no significant difference in 30-day mortality, septic shock and length of ICU stay between MRSA and MSSA infections. Similarly, HA-MRSA and CAMRSA developed comparable rates of septic shock, acute kidney injury (AKI) and 30-day mortality. However, cases of HA- S. aureus infections had more rates of AKI compared to CA-S. aureus cases. We observed this increased rate of AKI in HA-MSSA compared to CAMSSA infections.

In this study, the mortality rate of S. aureus infection was low (4.9\%) compared to the rates observed in other studies (7-50\%) [14-18]. This low rate of mortality could be explained by the low rate of $S$. aureus bacteremia in this study compared to the rates in the other studies. Many studies have shown that $S$. aureus bacteremic cases have increased morality compared to non-bacteremic cases [19]. Cosgrove et al in a meta-analysis demonstrated similar mortality between MRSA and MSSA infections [5]. Those few studies showed a higher MRSA mortality rate in this meta-analysis have many confounders and they were conducted two decades ago, before the current improvement MRSA treatment $[20,21]$.

We found a higher rate of AKI in HA-MSSA than in CA-MSSA cases. This could be attributed to the higher rates of chronic diseases in HAMSSA than in CA-MSSA cases. However, it is less likely this the only explanation as we have adjusted for chronic diseases in our analysis. Besides, we did not observe a higher rate in AKI in HA-MRSA when compared to CA-MRSA cases. Another possible explanation for the higher AKI rates in HA-MSSA cases, HA-MSSA strains may have a direct effect on the kidneys [22,23].

Our finding of comparable mortality between HA-MRSA and CAMRSA have been shown in many studies [24,25]. This could be due to CA- MRSA and HA- MRSA have similar virulence factors. Despite
HA MRSA and CA MRSA isolates have different genes, now they are epidemiologically indistinguishable. Studies have shown CAMRSA isolates caused nosocomial infections and HA-MRSA caused community-acquired infections [26]. In contrast, Kempker et al. showed increased risk of death in persons with CA-MRSA bacteremia due to USA300 MRSA strains compared to non-USA300 strains [27]. This study lack generalizability as it included high numbers of HIVinfected and intravenous drug users cases.

This study provides a new perspective on outcome of $S$. aureus infections and it has several implications. Our findings of MSSA and MRSA infections cause comparable outcomes. Emphasize that MSSA infections require prevention strategies similar to MRSA. The current hospital infection control interventions for MRSA such as surveillance programs, isolation precautions, and decolonization protocols may also help to interrupt MSSA transmission. Future research is need to identify HA-MSSA virulence factors, and to examine the effectiveness MRSA intervention to prevent MSSA infection.

Our study has several limitations. First, we may have underestimated the number of HA-S. aureus cases if its risk factors were not documented in the medical records. Yet, it is unlikely this influenced our findings, as our results are similar to the results of the studies used molecular genotyping to classify HA-S. aureus infections [24,26]. Second, in this study we reported all-cause mortality rather than attributable mortality, as the latter is difficult to determine. Third, we could not collect complete data on antimicrobial treatment. Therefore, we could not assess its potential association with $S$. aureus outcomes. However, we attempted to limit the effect of potential confounders by adjusting for infection severity and chronic comorbidities in our analysis.

\section{Conclusion}

In the present article, we investigated the outcome of community and hospital-acquired $S$. aureus infection. The results suggested that MRSA and MSSA have the similar clinical outcomes. Likewise, CAMRSA and HA-MRSA have a comparable 30-day morality. However, 
HA-MSSA associated with more cases of AKI compared to CA-MSSA infections. Taken together, this offers a new perspective on HA-MSSA as a serious pathogen as MRSA, that requires similar attention and intervention to prevent its spread. Future research may extend this work to identify additional measures for MSSA prevention and investigate whether current MRSA prevention strategies are also effective in MSSA prevention.

\section{Acknowledgement}

None.

\section{Conflict of Interest}

Author has no conflict of interest.

\section{References}

1. Styers D, Sheehan DJ, Hogan P, Sahm DF (2006) Laboratory-based surveillance of current antimicrobial resistance patterns and trends among Staphylococcus aureus: status in the United States. Ann Clin Microbiol Antimicrob 5: 2 .

2. Filice G, Nyman J, Lexau C, Christine H Lees, Lindsay A Bockstedt, et al. (2010) Excess Costs and Utilization Associated with Methicillin Resistance for Patients with Staphylococcus aureus Infection. Infect Control Hosp Epidemiol 31(4): 365-373.

3. Diep BA, Otto M (2008) The role of virulence determinants in community-associated MRSA pathogenesis. Trends Microbiol 16: 361-369.

4. Melzer M, Eykyn SJ, Gransden WR, Chinn S (2003) Is methicillin-resistant Staphylococcus aureus more virulent than methicillin-susceptible $S$. aureus? A comparative cohort study of British patients with nosocomial infection and bacteremia. Clin Infect Dis 37: 1453-1460.

5. Cosgrove SE, Sakoulas G, Perencevich EN, Schwaber MJ, Karchmer AW, et al. (2003) Comparison of mortality associated with methicillin-resistant and methicillin-susceptible Staphylococcus aureus bacteremia: a meta-analysis. Clin Infect Dis 36: 53-59.

6. Self WH, Wunderink RG, Williams DJ, Yuwei Zhu, Evan J Anderson, et al. (2016) Staphylococcus aureus Community-acquired Pneumonia: Prevalence, Clinical Characteristics, and Outcomes. Clin Infect Dis 63: 300-309.

7. Wyllie DH, Crook DW, Peto TE (2006) Mortality after Staphylococcus aureus bacteraemia in two hospitals in Oxfordshire, 1997-2003: cohort study. BMJ 333: 281

8. Fowler VG Jr, Olsen MK, Corey GR, Christopher W Woods, Christopher H Cabell, et al. (2003) Clinical identifiers of complicated Staphylococcus aureus bacteremia. Arch Intern Med 163: 2066-2072.

9. Willcox PA, Rayner BL, Whitelaw DA (1998) Community-acquired Staphylococcus aureus bacteremia in patients who do not abuse intravenous drugs. QJM 91: 41-47.

10. Joseph M Mylotte, Ammar Tayara (2003) Staphylococcus aureus Bacteremia: Predictors of 30-Day Mortality in a Large Cohort, Clinical Infectious Diseases. X 31: 1170-1174.

11. (2018) CLSI Performance Standards for Antimicrobial Susceptibility Testing. (28 $8^{\text {th }}$ edn.), CLSI supplement M100. Clinical and Laboratory Standards Institute, Wayne, PA, USA.
12. Fridkin SK, Hageman JC, Morrison M, Laurie Thomson Sanza, Kathryn Como-Sabetti, et al. (2005) Active Bacterial Core Surveillance Program of the Emerging Infections Program Network. Methicillin-resistant Staphylococcus aureus disease in three communities. N Engl J Med 352: 1436-1444.

13. Stavem K, Hoel H, Skjaker SA, Haagensen R (2017) Charlson comorbidity index derived from chart review or administrative data: agreement and prediction of mortality in intensive care patients. Clin Epidemiol 9: 311-320.

14. Levine DP, Crane LR, Zervos MJ (1986) Bacteremia in narcotic addicts at the Detroit Medical Center. II. Infectious endocarditis: a prospective comparative study. Rev Infect Dis 8: 374-96.

15. Lewis E, Saravolatz LD (1985) Comparison of methicillin-resistant and methicillin-sensitive Staphylococcus aureus bacteremia. Am J Infect Control 13: 109-114.

16. Roghmann MC (2000) Predicting methicillin resistance and the effect of inadequate empiric therapy on survival in patients with Staphylococcus aureus bacteremia. Arch Intern Med $160: 1001-4$.

17. Selvey LA, Whitby M, Johnson B (2000) Nosocomial methicillin-resistant Staphylococcus aureus bacteremia: is it any worse than nosocomial methicillin-sensitive Staphylococcus aureus bacteremia? Infect Control Hosp Epidemiol 21: 645-648.

18. Soriano A, Martinez JA, Mensa J, M T Jiménez de Anta, E Soriano, et al (2000) Pathogenic significance of methicillin resistance for patients with Staphylococcus aureus bacteremia. Clin Infect Dis 30: 368-373.

19. 1 bloodstream infections on patient outcomes in the ICU setting. Chest 118: 146-155.

20. Jackson KA, Gokhale RH, Nadle J, William Schaffner, David C Ham, et al. (2020) Public Health Importance of Invasive Methicillin-sensitive Staphylococcus aureus Infections: Surveillance in 8 US Counties, 2016. Clin Infect Dis 70: 1021-1028.

21. Gerald L Mandell, John E Bennett, Raphael Dolin Mandell (2009) Douglas and Bennett's principles and practice of infectious diseases. ( $7^{\text {th }}$ edn.), pp. 1077.

22. Koyama A, Sharmin S, Sakurai H, Keigyou Yoh, Kunihiro Yamagata, et al. (2004) Staphylococcus aureus cell envelope antigen is a new candidate for the induction of IgA nephropathy Kidney International 66: 121-132.

23. Popovich KJ, Weinstein RA, Hota B (2008) Are community-associated methicillin-resistant Staphylococcus aureus (MRSA) strains replacing traditional nosocomial MRSA strains? Clin Infect Dis 46: 787-794.

24. Nair R, Ammann E, Rysavy M, Schweizer ML (2014) Mortality among patients with methicillin-resistant Staphylococcus aureus USA300 versus non-USA300 invasive infections: a meta-analysis. Infect Control Hosp Epidemiol 35: 31-41.

25. Popovich KJ, Snitkin ES, Hota B, Stefan J Green, Ali Pirani, et al. (2017) Genomic and Epidemiological Evidence for Community Origins of Hospital-Onset Methicillin-Resistant Staphylococcus aureus Bloodstream Infections. J Infect Dis 215: 1640-1647.

26. Kempker RR, Farey MM, Ladson JL, Sarah Satola, Susan M Ray, et al. (2010) Association of methicillin-resistant Staphylococcus aureus (MRSA) USA300 genotype with mortality in MRSA bacteremia. J Infect 61: $372-381$ 